\title{
Cinémas
}

Revue d'études cinématographiques

Journal of Film Studies

\section{ALBERSMEIER, Franz-Josef. Theater, Film, Literatur in Frankreich. Medienwechsel und Intermedialitat. Darmstadt : Wissenschaftliche Buchgesellschaft, 1992}

\section{Jürgen E. Müller}

Volume 5, numéro 1-2, automne 1994

URI : https://id.erudit.org/iderudit/1001015ar

DOI : https://doi.org/10.7202/1001015ar

Aller au sommaire du numéro

Éditeur(s)

Cinémas

ISSN

1181-6945 (imprimé)

1705-6500 (numérique)

Découvrir la revue

Citer ce compte rendu

Müller, J. E. (1994). Compte rendu de [ALBERSMEIER, Franz-Josef. Theater,

Film, Literatur in Frankreich. Medienwechsel und Intermedialitat. Darmstadt :

Wissenschaftliche Buchgesellschaft, 1992]. Cinémas, 5(1-2), 223-228.

https://doi.org/10.7202/1001015ar d'utilisation que vous pouvez consulter en ligne. 
ALBERSMEIER, Franz-Josef. Theater, Film, Literatur in Frankreich. Medienwechsel und Intermedialitat. Darmstadt : Wissenschaftliche Buchgesellschaft, 1992

L'état des recherches cinématographiques peut être caractérisé par deux réductions fondamentales: a) à ses débuts, la discipline a développé la tendance à se séparer d'une manière très claire des disciplines voisines (soit les "lettres", soit les "sciences sociales") et à se définir sur la base de cette séparation; b) les études cinématographiques ont très souvent restreint leurs recherches à un seul média isolé (au "film", bien sûr) ou même à un seul aspect de ce média, c'est-à-dir à l'image cinématographique. C'est le cas dans l'histoire et dans la théorie du film.

Naturellement, il faut bien rester conscient du fait que des activités scientifiques impliquent inévitablement certaines réductions par rapport aux "objets" analysés, mais aujourd'hui on peut avoir l'impression que la "crise des études cinématographiques" (surtout déplorée dans le contexte anglo-saxon) est, entre autres, un des résultats de ces réductions traditionnelles, et qui doivent être surmontées.

Ce sont justement ces réductions contre lesquelles Franz-Josef Albersmeier se prononce dans son livre. Il nous avertit de la nécessité de changer la pratique scientifique de l'historiographie des médias isolés. Chacun de nous connaît la multitude d'histoires du théâtre, du film, de la littérature, où les médias sont traités commes des monades médiatiques. Mais devant ce grand nombre d'histoires réductrices de films nationaux ou de médias se trouvent très peu d'études qui décriraient l'interdépendance et l'interaction historique des différents médias.

Si cet argument d'Albersmeier est correct, il faut en tirer les conséquences et écrire, par exemple, une histoire théatrale et 
filmique de la littérature, une histoire filmique et littéraire du théatre, ou une histoire littéraire et théattrale du film - pour ne nommer que les options les plus évidentes. (Et il ne faut pas oublier le fait qu'il y a encore beaucoup d'autres médias dans ces processus historiques.) La conception d'une telle histoire intégrée - et très complexe, bien sûr - des médias est donc

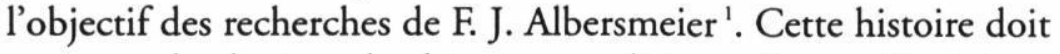
surpasser les limites des histoires traditionnelles et elle doit se présenter sous une autre forme que les simples énumérations de sujets ou de techniques médiatiques, utilisés dans tel ou tel média; elle doit se diriger sur les interactions médiatiques qui ont lieu sur différents niveaux textuels et institutionnels. La procédure historiographique suggérée dans Theater, Film, Literatur in Frankreich sera donc une approche inductive qui permettra de re-construire les mécanismes principaux de ces interactions. Sur ce point, le livre d'Albersmeier est un premier pas dans une bonne direction et sur un terrain scientifique inconnu où, jusqu'à maintenant, nous ne pouvions guère trouver de coordonnées.

Comme le titre du livre l'indique, Albersmeier base son histoire intégrée sur un corpus de productions culturelles françaises - et c'est dès les premières pages que le lecteur a l'impression que l'auteur a fait un bon choix, en nous rappelant le fait que dans l'histoire des (relations des) médias, la culture française a joué un rôle exceptionnel, par rapport au développement de structures intermédiatiques dans les œuvres d'art et par rapport aux relations intermédiatiques au cours de l'histoire des différents médias. Pour donner un exemple: la tradition française du scénario et des scénaristes se caractérise par des transgressions permanentes de limites médiatiques. Des écrivains deviennent des "écrivains scénaristes", des directeurs de films, des "écrivains cinéastes", certains directeurs sont à la fois des scénaristes sous forme d' "écrivain-scénariste-cinéaste" (p. 151). Et ces transgressions ont des effets multiples sur la production des différents " textes médiatiques".

Albersmeier approche ces phénomènes sur la base très riche de sources et de matériaux (filmiques, théâtraux et littéraires) qu'il a rassemblés. La complexité des processus exige une 
réduction scientifique et conséquemment, il fait porter ses recherches sur cinq différents types d'interaction entre trois médias principaux du $\mathrm{XX}^{e}$ siècle. Le premier chapitre traite des "interactions entre théâtre et film". (C'est le théâtre "contre" le film, et le film "contre" le théâtre.) Le deuxième vise "l'avantgarde littéraire et le film ", le troisième, la "transgression médiatique avec ou sans passage entre des médias ", le quatrième, "l'adaptation filmique de textes littéraires" et le cinquième, "des aspects du film littéraire et théâtral en France".

En regardant les titres de ces chapitres, le caractère ambitieux de l'entreprise devient évident. Et si nous comparons ces titres au contenu du livre, on ne peut que féliciter Albersmeier de la profondeur et de la solidité de ses résultats de recherche qui sont convaincants.

Mais, ne l'oublions pas, Theater, Film, Literatur in Frankreich commence avec une digression: ce sont les jeux entre "caméra " et "stylo", c'est-à-dire "les relations entre la littérature et la photographie du XIX siècle" qui constituent un premier pas vers l'esquisse d'une histoire intégrée. Et dans ce chapitre très condensé, le lecteur ne trouve pas les courts-circuits du discours scientifique traditionnel. Albersmeier démontre que l'influence de la photographie sur la littérature française (surtout "réaliste" et "naturaliste") doit d'abord être conçue comme influence intertextuelle de certains répertoires de textes avant d'être considérée comme une influence de certaines structures médiatiques. Autrement dit, malgré les références théoriques à la photographie d'un Balzac, par exemple, malgré cette forme de visualisation croissante dans l'œuvre de Flaubert (de Madame Bovary à L'Éducation sentimentale) et malgré les efforts assez tardifs de Zola à utiliser des photographies comme des documents de travail pour son œuvre naturaliste, on ne peut pas constater un "développement continuel dans le sens d'une représentation fictive de la réalité qui soit de plus en plus pénétrée de la photographie" (p. 21). Néanmoins, vers la fin du XIX éècle, la photographie gagne une valeur toujours plus importante pour les écrivains qui commencent à reconnaître l'indépendance de ce média, qui devient alors le point de départ de "nouvelles" interactions médiatiques entre littérature et photographie — des 
interactions qui se manifestent entre autres dans des textes " multimédiatiques " comme Nadja de Breton (p. 140-149).

Après cette digression introductrice - et bien utile d'ailleurs —, Albersmeier aborde le développement historique des relations entre théâtre, film et littérature en France. Il a raison d'être prudent et de ne pas présenter des conclusions théoriques précipitées en ayant délibérément choisi le chemin pénible d'une reconstruction de constellations et de structures historiques sur la base des "faits" qu'il a pu trouver dans les différents textes médiatiques (p. 57). Et c'est cette approche qui produit une richesse impressionnante de résultats (par exemple, sur les "cas" Antoine du "théâtre cinéma", Pagnol du "cinéma théâtre " Artaud du "théâtre filmique".

Il me semble tout de même utile d'ajouter ici une ou deux remarques théoriques. Étant donnée la complexité des processus intermédiatiques, on peut bien suivre l'argument d'Albersmeier de vouloir faire naître une idée théorique du concept de l'intermédialité à travers la re-construction historique et détaillée de ces processus. Mais d'un autre côté, il serait souhaitable que le lecteur puisse trouver quelques commentaires de l'auteur sur les suggestions ou les définitions qui existent déjà en ce moment ${ }^{2}$.

Une telle discussion (même courte) serait aussi utile afin de pouvoir effectuer une différenciation au sein de la classification des médias suggérée par Albersmeier, différenciation qui serait à conseiller pour les recherches futures. Si l'on considère "le film" comme média "électronique" (comme cela est proposé dans le livre), on exclut per definitionem un grand nombre de processus intermédiatiques qui jouent justement avec la différence entre l'ancien média "chimio-mécanique ${ }^{3}$ " (qui a bien sûr changé pendant les années passées) et les médias "électroniques" de la télévision, de la vidéo, de HDTV, etc. ${ }^{4}$ Un écran de télévision est bien différent d'un écran de cinéma, et c'est le même pour leurs images qui oscillent entre le "ça peut être" d'images artificielles et digitales de l'art vidéo, par exemple, et le "ça a étés" du film. Sans une telle différenciation, une histoire intégrée des médias devrait supprimer tous les processus partagés entre film, radio, télévision et (l'art-)vidéo ainsi que leur rôle important dans nos temps (post?)modernes, puis nous n'aurions plus les moyens 
d'étudier, par exemple, Passion de Godard ou Prospero's Books de Greenaway. Car ce sont justement les interactions entre l'ancien dispositif du film et les procédés électroniques et digitaux des nouveaux médias qui écrivent un nouveau chapitre dans une histoire intégrée de l'audiovisuel.

Mais revenons donc à Theater, Film, Literatur in Frankreich. Le grand mérite de ce livre consiste dans la suppression d'anciennes pistes scientifiques à sens unique et qui sont élargies en des pistes à deux (ou même plusieurs) sens. Albersmeier ne nous montre pas seulement les processus d'adaptation filmique de textes littéraires à l'exemple de films choisis de René Clair, Resnais, Rappenau, Renoir, Gervaise et Truffaut (p. 185 et suivantes) sous la nouvelle perspective de son approche, mais nous fait voir aussi l'" autre direction" de ces processus, c'est-àdire l'intrusion du filmique "dans" la littérature à l'exemple de l'avant-garde française (Appollinaire, Aragon, Cendrars, Breton, p. 81 et suivantes).

Il nous fait voir comment les jeux multi- et intermédiatiques entre et dans des "genres bâtards" comme le "ciné-roman " des années dix et vingt ont contribué à des histoires intégrées de production et de réception des deux médias - des histoires qui, à l'instant, ne sont guère connues ou analysées (p. 100 et suivantes).

Le dernier chapitre du livre se concentre sur les aspects principaux du film littéraire-théatral en France et une fois de plus nous apprenons à l'exemple de trois films (L'Assassinat du duc de Guise [1908], Les Enfants du paradis et Les jeux sont faits) quelques règles des jeux historiques et intermédiatiques entre film, thêâtre et littérature.

L'ouvrage conclut avec deux "chapitres" complémentaires très utiles (une liste de films de "documentaires littéraires" et des renseignements pratiques sur des cinémathèques, éditeurs de scénarios, librairies et bibliographies sur le film) et avec une bibliographie et un index très riches.

Arrivé à la fin de mon court compte rendu, je n'ai qu'à formuler, en accord avec Albersmeier, que l'on ne peut pas attendre d'un tel projet d'une histoire "intégrée " des médias des formules ou des règles fixes sous forme de conclusions générales 
pour "tous" les processus intermédiatiques (p. 243) entre film, théâtre et littérature. Mais, bien sûr, on trouve une première différenciation historique et très stimulante entre les phénomènes complexes, qui exige encore beaucoup de recherches futures - de même que pour les autres médias audiovisuels de notre siècle.

JƯRGEN E. MƯLLER

Université d'Amsterdam

\section{NOTES}

1 Theater, Film, Literatur in Frankreich n'est pas le seul ni le premier livre d'Albersmeier sur ce sujet. Je rappelle ici les ouvrages suivants: André Malraux und der Film. Zur Rezeption des Films in Frankreich (Berlin/Frankfurt: Lang, 1973), Bild und Text. Beitrage zu Film und Literatur (1976-1982) (Bern/Frankfurt: Lang, 1983) et Die Herausforderung des Films an die franzosische Literatur. Entwurf einer "Literaturgeschichte des Films", I: Die Epoche des Stummfilms (1895-1930) (Heidelberg: Carl Winter, 1985).

2 Des suggestions et définitions qu'on retrouve dans les articles et les livres suivants: Jacques Aumont, L'Eil interminable. Cinéma et peinture (Paris: Séguier, 1989); Claus Cluver, "Reflections on Verbivocovisual Ideograms", Poetics Today, vol. 3, $\mathrm{n}^{\circ} 3$ (1982) p. 137-148; Dick Higgins, Horizons. The Poetics and Theory of the Intermedia (Carbondale / Edwardsville: Southern Illinois University Press, 1984); Karl Prümm, "Multimedialitat und Intermedialitat ", Theaterzeitschrift, H. 22, IV (1987) p. 95-103; Aage Hansen-Love, "Worter und/oder Bilder. Probleme der Intermedialitat mit Beispielen aus der russischen Avantgarde", Eikon. Internationale Zeitschrift fur Photographie ơ Medienkunst, H. 4 (1992) p. 32-41 ; Jürgen E. Müller, "Intermedialitat als Provokation der Medienwissenschaft", Eikon. Internationale Zeitschrift fur Photographie of Medienkunst, H. 4 (1992) p. 13-21.

3 Je trouve la fameuse thèse de Marshall Mc Luhan, selon laquelle le film serait le dernier média du XIX ${ }^{e}$ siècle et le premier du XXé, toujours convaincante. Selon cette thèse, le film se caractérise par une combinaison du mécanique du XIX siècle avec l'électricité. Et malgré toutes les options que les nouvelles technologies lui offrent aujourd'hui, l'" accélération du mécanique" restera toujours un des traits caractéristiques du dispositif (Pour comprendre les média, Paris: Seuil, 1976, p. 30).

4 Voir Raymond Bellour, L'Entre-Images. Photo. Cinéma. Vidéo (Paris: La Différence, 1990); Florian Rotzer (direction) Digitaler Schein. Asthetik der elektronischen Medien (Frankfurt: Suhrkamp, 1991); et aussi, mon livre à paraître, Intermedialitat: Formen moderner kultureller Kommunikation (Münster : Nodus, 1994).

5 Voir Edmond Couchot, Images. De l'optique au numérique (Paris: Hermès, 1988) et le compte rendu de Bernard Bérubé, Cinémas, vol. 1, n 3 (1991) p. 146150. 